\title{
Software Reliability Modelling Considering both Testing Effort and Testing Coverage
}

\author{
Liu Chang ${ }^{1}$, Liu Yuan ${ }^{2}$, Ren Zhanyong ${ }^{1}$, Li Haifeng ${ }^{1}$ \\ ${ }^{1}$ China Aero-Polytechnology Establishment, BeiJing 100028, China \\ ${ }^{2}$ The Air Force Military Representative Office in JingChang Area, \\ Beijing 100091, China \\ email:yongxin765@126.com
}

\begin{abstract}
.
Considering testing effort function (TEF) or testing coverage function (TCF) in the non-homogeneous Poisson process (NHPP) software reliability modeling can further improve the fitting and prediction performance of the NHPP software reliability growth models (SRGMs). Thus this paper discusses how to integrate both TEF and TCF into the traditional NHPP software reliability modeling process to capture the integrated effect of testing effort and testing coverage on reliability estimation. First we present a new TEF, i.e. the inflected S-shaped TEF (IS-TEF) for describing the S-shaped varying trend of the testing-effort increasing rate more accurately. Meanwhile, a new NHPP SRGM (named IS-SRGM) which considers the IS-TEF is proposed. Then a comprehensive modeling framework for incorporating the TEF and TCF is proposed. Recur to this framework, a new NHPP SRGM (named IS-LO-SRGM) with both the IS-TEF and logistic TCF (LO-TCF) is proposed. Finally, two case studies for validating the effectiveness of the IS-TEF and the IS-LO-SRGM in terms of fitting results are presented. The results show that: the comprehensive modeling framework to incorporate the TEF and the TCF in NHPP SRGMs achieves a substantial improvement compared with the NHPP SRGMs which only consider the TEF or the TCF.

Keywords: Software Reliability Modeling, NHPP, Testing Effort, Testing Coverage
\end{abstract}

\section{Introduction}

As the main approaches for software reliability estimation and prediction, many time-related SRGMs have been proposed for the safety-critical software [1-3]. Especially, the NHPP SRGMs may be the most well-known SRGMs due to their mathematical tractability [4]. Testing effort indicates the consumed resources during testing phase and can be measured as human power, the number of test cases, the number of CPU hours and etc [8-10]. As a result, several new NHPP SRGMs were proposed by incorporating diverse testing effort functions (TEFs) into the traditional NHPP modeling process, such as the exponential, 
Rayleigh, Weibull [13], exponentiated Weibull [14], Burr-type X [15], logistic [9], generalized logistic $1 \& 2$ [16] TEFs and so on.

Similarly, testing coverage which can be measured in terms of blocks, branches, c-uses, p-uses that are covered is a good indicator for testing completeness and effectiveness [12]. Testing coverage has been proved that it has certain effects on software reliability estimation [17-18]. Thus, several NHPP SRGMs were proposed by incorporating various testing coverage functions (TCFs), such as the exponential, Weibull, S-shaped, log-logistic [19], Pham-Zhang [20], logistic [21] TCFs.

In this paper, we will elucidate how to integrate both TEF and TCF into the traditional NHPP SMGM. The rest of this paper is organized as follows. In Section 2, we propose a new S-shaped TEF, i.e. the IS-TEF and then introduce a S-shaped TCF, i.e. the LO-TCF. In Section 3, we present a new NHPP SRGMs considering the IS-TEF. In Section 4, we propose a comprehensive modeling framework for incorporating the TEF and TCF together. A new NHPP SRGMs is developed by taking the IS-TEF and LO-TCF into the modeling framework. Finally, a case study is presented for validating the fitting performance of the proposed NHPP SRGMs.

\section{Inflected S-shaped TEF and Logistic TCF}

1. Inflected S-shaped TEF (IS-TEF)

We use the inflected S-shaped function to describe the expenditure pattern of actual testing-effort data. Then the cumulative amount of testing-effort consumed in $(0, t)$ can be depicted as follows:

$$
W(t)=W_{\max } \frac{1-e^{-b t}}{1+\psi e^{-b t}}
$$

$$
\text { According to Eq.1, we have: } w(t)=\frac{d W(t)}{d t}=\frac{W_{\max } b \exp (-b t)(1+\psi)}{(1+\psi \exp (-b t))^{2}}
$$

\section{Logistic TCF (LO-TCF)}

In our previous study [21], we utilized the logistic function to describe the S-shaped growth curve of testing time. The logistic TCF is shown as follows: $C(t)=\frac{C_{\max }}{1+A \exp (-\alpha t)}$

$$
\begin{gathered}
\text { For convenience, we suppose } \quad \mathrm{C}_{\max } \quad \text { equals } \quad 100 \% \quad \text { and } \\
\text { then: } C(t)=\frac{1}{1+A \exp (-\alpha t)}
\end{gathered}
$$




\section{Software reliability modelling with inflected S-shaped TEF}

The modeling framework of the NHPP SRGM with various TEFs can be shown as follows:

$$
\frac{d m(t)}{d t} \times \frac{1}{w(t)}=r(W(t))[N-m(t)]
$$

Solving the above differential equation under the boundary condition $\mathrm{m}(0)=0$ and $\mathrm{W}(0)=0$, then the exponential NHPP SRGM with IS-TEF (denoted by IS-TEFM in this paper) is shown as:

$$
m(t)=N\left\{1-\exp \left[-r W_{\max } \frac{1-\exp (-b t)}{1+\psi \exp (-b t)}\right]\right\}
$$

\section{Software reliability modeling considering TEF and TCF}

The modeling framework of the SRGM with TEF and TCF is shown as follows:

$$
\frac{d m(t)}{d t} \frac{1}{w(t)}=\frac{C^{\prime}(W(t))}{1-C(W(t))}[N-m(t)]
$$

A mean value function as the solution of the differential equation in Eq.7 can be given as follows with the boundary condition $\mathrm{m}(0)=0$ according to [20]

$$
\begin{array}{r}
m(t)=N \mathrm{e}^{-B(W(t))} \int_{0}^{t} \mathrm{e}^{B(W(\tau))} \frac{C^{\prime}(W(\tau))}{1-C(W(\tau))} d \tau \\
\left.B(t)=\int_{0}^{t} \frac{C^{\prime}(W(\tau))}{1-C(W(\tau))} d \tau\right)
\end{array}
$$

First, integrating the LO-TCF into the comprehensive modeling framework (Eq.8), we have

$$
\frac{d m(t)}{d t} \frac{1}{w(t)}=\frac{\alpha(N-m(t))}{1+A \exp (-\alpha W(t))}
$$

Then taking the IS-TEF into the above differential equation, and solving the Eq.9 under the boundary condition $\mathrm{m}(0)=0$, a new NHPP SRGM (denoted by IS-LO-SRGM) with both IS-TEF and LO-TCF can be obtained and shown as follows: 


$$
m(t)=N\left[1-\exp \left(-\frac{\alpha W_{\max } \frac{1-e^{-b t}}{1+\psi e^{-b t}}}{1+A \exp \left(-\alpha W_{\max } \frac{1-e^{-b t}}{1+\psi e^{-b t}}\right)}\right)\right]
$$

\section{Case Studies}

Two real failure data-sets concluding the testing-effort data are selected for case studies. The first data-set (DS1) was collected by Ohba. We also select six various representative NHPP SRGMs (shown in Tab.2) as the comparison models for case study 2, namely the Goel-Okumoto model (GO) [3], Inflected S-shaped model (IS), Logistic-TCF model (LOTCFM) [21], Pham-Zhang-TCF model (PZTCFM) [20], Logistic-TEF model (LOTEFM) [9], and exponentiated-Weibull-TEF model (EWTEFM) [14]. Two comparison criteria are selected for comparing the fitting power:

1) The Mean Square Error (MSE) [5]: $\quad \operatorname{MSE}=\frac{1}{n} \sum_{i=1}^{n}\left(m\left(t_{i}\right)-m_{i}\right)^{2}$ (11) where $m_{i}$ is the observed number of faults at $t_{i}, m\left(t_{i}\right)$ is the estimated value of the number of faults at $t_{\mathrm{i}}$. The lower the MSE is, the better the SRGM fits.

2) The Akaike Information Criterion (AIC) [4]: AIC $=-2 * \mathrm{MLL}+2 * N_{p}$ (12)

The lower the AIC is, the better the SRGM fits.

The estimated parameters and the fitting results of these comparison NHPP SRGMs for each data-set are shown in Tab.1. The bold number indicates the best fitting result in this column.

Tab.1 The Fitting Results of the NHPP SRGMs in Case Study

\begin{tabular}{|c|c|c|c|c|c|c|}
\hline \multirow[b]{2}{*}{ SRGM } & \multicolumn{3}{|c|}{ DS1 } & \multicolumn{3}{|c|}{ DS2 } \\
\hline & Parameters & AIC & MSE & Parameters & AIC & $\begin{array}{c}\text { MS } \\
\text { E }\end{array}$ \\
\hline GO & $\begin{array}{l}N=513.15 \\
r=0.0537\end{array}$ & $\begin{array}{l}220.7 \\
6\end{array}$ & $\begin{array}{l}139.8 \\
2\end{array}$ & $\begin{array}{c}N=133.43 \\
r=0.045\end{array}$ & 120.93 & 22.9 \\
\hline IS & $\begin{array}{c}N=355.06 \\
r=0.222 \\
\psi=3.27\end{array}$ & $\begin{array}{l}207.7 \\
2\end{array}$ & $\begin{array}{l}127.3 \\
1\end{array}$ & $\begin{array}{c}N=88.606 \\
r=0.249 \\
\psi=6.186\end{array}$ & 111.06 & 5.10 \\
\hline LOTCFM & $\begin{array}{c}N=361.715 \\
3 \\
A=2.3199 \\
\alpha=0.1438\end{array}$ & $\begin{array}{c}206.2 \\
6\end{array}$ & 86.05 & $\begin{array}{c}N=89.27 \\
9 \\
A=4.229 \\
\alpha= \\
0.1665\end{array}$ & 110.86 & 5.17 \\
\hline
\end{tabular}




\begin{tabular}{|c|c|c|c|c|c|c|}
\hline PZTCFM & $\begin{array}{l}N=519.61 \\
\beta=0.0097 \\
b=0.1982\end{array}$ & $\begin{array}{c}218.4 \\
4\end{array}$ & $\begin{array}{l}138.6 \\
9\end{array}$ & $\begin{array}{c}N=161.06 \\
\beta=0.0127 \\
b=0.158\end{array}$ & $\begin{array}{c}109.97 \\
0\end{array}$ & 8.85 \\
\hline LOTEFM & $\begin{array}{c}N=351.09 \\
r=0.0344\end{array}$ & $\begin{array}{c}213.7 \\
3\end{array}$ & 114.05 & $\begin{array}{c}N= \\
92.7942 \\
r=0.0298\end{array}$ & 110.581 & 5.88 \\
\hline EWTEFM & $\begin{array}{c}N=568.54 \\
r=0.0194\end{array}$ & $\begin{array}{c}216.8 \\
5 \\
\end{array}$ & 113.68 & $\begin{array}{c}N=94.7240 \\
r=0.0254\end{array}$ & $\begin{array}{c}108.74 \\
1 \\
\end{array}$ & 7.05 \\
\hline IS-TEFM & $\begin{array}{c}N=554.05 \\
r=0.0201\end{array}$ & $\begin{array}{l}216.1 \\
6 \\
\end{array}$ & $\begin{array}{l}107.0 \\
5 \\
\end{array}$ & $\begin{array}{c}N=110.49 \\
r=0.0274\end{array}$ & 116.461 & 5.28 \\
\hline $\begin{array}{c}\text { IS-LO-SRG } \\
\mathrm{M} \\
\end{array}$ & $\begin{array}{c}N=378.759 \\
A=1.21 \\
\alpha=0.051\end{array}$ & $\begin{array}{l}205.6 \\
0\end{array}$ & 83.05 & $\begin{array}{c}N=94.69 \\
A=0.081 \\
\alpha=0.0261\end{array}$ & 111.122 & 5.01 \\
\hline
\end{tabular}

For the DS1 and DS2, the proposed IS-LO-SRGM yields the best fitting results. In other words, for these two data-set, the IS-LO-SRGM not only has the lowest value of AIC, but also has the lowest value of MSE. Consequently, it indicates that the IS-LO-SRGM has the best fitting performance compared with the comparison SRGMs.

\section{Conclusions}

Testing effort and testing coverage are believed to be two most important testing factors which can be considered in reliability modeling process for further improving the fitting and prediction power of the traditional NHPP SRGMs. Thus this paper discusses how to integrate both testing effort function and testing coverage function into the traditional NHPP software reliability modeling process based on the NHPP software reliability modeling framework considering the TEF. The experimental results show that: the IS-LO-SRGM considering both IS-TEF and LO-TCF nearly yields the best fitting results compared with the other comparison NHPP SRGMs for two data-sets.

\section{Acknowledgement}

This paper is supported by the technology research project of Technology and Industry for National Defence Z052013B009.

\section{References}

[1] Lyu, M. R. Handbook of Software Reliability Engineering. USA: McGraw Hill; 1996

[2] Kapur, P. K., Ompal, S., Aggarwal, A. G., Kumar, R. Unified Framework for Developing testing Effort Dependent Software Reliability Growth Models. WSEAS Transactions on Systems 2009; 4(8): 521-531 
[3] Pham, H. Software reliability. Germany: Springer-Verlag; 2000

[4] H. Okamura, Y. Etani, T. Dohi. A multi-factors Software reliability model based on Logistic regression. In: IEEE 21st International Symposium on Software Reliability Engineering (ISSRE). Washington DC: IEEE Computer Society; 2010: 31-40

[5] Huang, C. Y., Kuo, S. Y., Lyu, M. R. An assessment of testing-effort dependent software reliability growth models. IEEE Transactions on Reliability 2007; 56(2): 198-211

[6] K. Shibata, K. Rinsaka, T. Dohi. Metrics-based software reliability models using non-homogeneous Poisson processes. In: Proceedings of IEEE International Symposium on Software Reliability Engineering (ISSRE). Washington DC: IEEE Computer Society; 2006: 52-61

[7] Cai, X., Lyu, M. R. Software Reliability Modeling with Test Coverage Experimentation and Measurement with a Fault-Tolerant Software Project. In: ISSRE. Washington DC: IEEE Computer Society; 2007:17-26

[8] Kuo, S. Y., Huang, C. Y., Lyu, M. R. Framework for modeling software reliability, using various testing-efforts and fault-detection rates. IEEE Transactions on Reliability 2001; 50(3): 310-320

[9] Huang, C. Y., Kuo, S. Y. Analysis of incorporating logistic testing-effort function into software reliability modeling. IEEE Trans. on Reliability 2002; 51(3): 261-270

[10] Ahmad, N., Khan and et al. A study of testing-effort dependent inflection S-shaped software reliability growth models with imperfect debugging. International Journal of Quality \& Reliability Management 2010; 27(1): 89-110

[11] Norman, E. F., Martin, N. A critique of software defect prediction models. IEEE Trans. on Software Engineering 1999; 25(5): 675-688

[12] Yashwant K., Malaiya, Michael Naixin Li, "Software reliability growth with test coverage”, IEEE Transactions on Reliability, 2002, 51(4): 420 426

[13] Yamada, S., Hishitani, J., Osaki, S. Software reliability growth model with Weibull testing effort: a model and application. IEEE Transactions on Reliability 1993; 42(1): 100-106

[14] Ahmad, N., Bokhari, M. U., Quadri, S. M. K., Khan, M. G. M. The exponentiated Weibull software reliability growth model with various testing-efforts and optimal release policy. International Journal of Quality \& Reliability Management 2008; 25(2): 211-235

[15] Ahmad, N., Khan, M. G. M., Quadri, S. M. K., Kumar, M. Modeling and analysis of software reliability with Burr type $\mathrm{X}$ testing-effort and release-time determination. Journal of Modeling in Management 2009; 4(1): 28-54

[16] Huang, C. Y., Lyu, M. R. Optimal release time for software systems considering cost, testing-effort, and test efficiency. IEEE Trans. on Reliability 2005; 54(4): 583-591 